\title{
Article
}

\section{Observational signatures of outbursting protostars - I: From hydrodynamic simulations to observations}

MacFarlane, Benjamin, Stamatellos, Dimitris, Johnstone, Doug, Herczeg, Gregory, Baek, Giseon, Chen, Huei-Ru Vivien, Kang, SungJu and Lee, Jeong-Eun

Available at http://clok.uclan.ac.uk/28815/

MacFarlane, Benjamin, Stamatellos, Dimitris ORCID: 0000-0002-4502-8344, Johnstone, Doug, Herczeg, Gregory, Baek, Giseon, Chen, Huei-Ru Vivien, Kang, Sung-Ju and Lee, Jeong-Eun (2019) Observational signatures of outbursting protostars - I: From hydrodynamic simulations to observations. Monthly Notices of the Royal Astronomical Society, 487 (4). pp. 5106-5117. ISSN 00358711

It is advisable to refer to the publisher's version if you intend to cite from the work. http://dx.doi.org/10.1093/mnras/stz1512

For more information about UCLan's research in this area go to http://www.uclan.ac.uk/researchgroups/ and search for <name of research Group>.

For information about Research generally at UCLan please go to http://www.uclan.ac.uk/research/

All outputs in CLoK are protected by Intellectual Property Rights law, including Copyright law. Copyright, IPR and Moral Rights for the works on this site are retained by the individual authors and/or other copyright owners. Terms and conditions for use of this material are defined in the policies page. 


\title{
Observational signatures of outbursting protostars - I: From hydrodynamic simulations to observations
}

\author{
Benjamin MacFarlane, ${ }^{1}$ Dimitris Stamatellos ${ }^{\circledR},{ }^{1 \star}$ Doug Johnstone, ${ }^{2,3}$ \\ Gregory Herczeg, ${ }^{4}$ Giseon Baek, ${ }^{5}$ Huei-Ru Vivien Chen, ${ }^{6}$ Sung-Ju Kang ${ }^{7}$ and \\ Jeong-Eun Lee ${ }^{5}$ \\ ${ }^{1}$ Jeremiah Horrocks Institute for Mathematics, Physics and Astronomy, University of Central Lancashire, Preston PR1 2HE, UK \\ ${ }^{2}$ NRC Herzberg Astronomy and Astrophysics, 5071 West Saanich Rd, Victoria V9E 2E7, Canada \\ ${ }^{3}$ Department of Physics and Astronomy, University of Victoria, Victoria V8P 1A1, Canada \\ ${ }^{4}$ Kavli Institute for Astronomy and Astrophysics, Peking University, Yiheyuan 5, Haidian Qu, 100871 Beijing, China \\ ${ }^{5}$ School of Space Research and Institute of Natural Sciences, Kyung Hee University, 1732 Deogyeong-daero, Giheung-gu, Yongin-si, Gyeonggi-do 446-701, \\ Republic of Korea \\ ${ }^{6}$ Department of Physics \& Astornomy, National Tsing Hua University, Kuang Fu Road, Hsinchu, 30013 Taiwan \\ ${ }^{7}$ Korea Astronomy and Space Science Institute, 776 Daedeokdae-ro, Yuseong-gu, Daejeon 34055, Republic of Korea
}

Accepted 2019 May 16. Received 2019 April 27; in original form 2019 March 1

\begin{abstract}
Accretion onto protostars may occur in sharp bursts. Accretion bursts during the embedded phase of young protostars are probably most intense, but can only be inferred indirectly through long-wavelength observations. We perform radiative transfer calculations for young stellar objects (YSOs) formed in hydrodynamic simulations to predict the long wavelength, sub-mm and $\mathrm{mm}$, flux responses to episodic accretion events, taking into account heating from the young protostar and from the interstellar radiation field. We find that the flux increase due to episodic accretion events is more prominent at sub-mm wavelengths than at $\mathrm{mm}$ wavelengths; e.g. a factor of $\sim 570$ increase in the luminosity of the young protostar leads to a flux increase of a factor of 47 at $250 \mu \mathrm{m}$ but only a factor of 10 at $1.3 \mathrm{~mm}$. Heating from the interstellar radiation field may reduce further the flux increase observed at longer wavelengths. We find that during FU Ori-type outbursts the bolometric temperature and luminosity may incorrectly classify a source as a more evolved YSO due to a larger fraction of the radiation of the object being emitted at shorter wavelengths.
\end{abstract}

Key words: accretion, accretion discs - radiative transfer-stars: protostars - stars: variables: general .

\section{INTRODUCTION}

The mass growth of a protostar is expected to occur primarily during the initial phases of its formation while it is still embedded in its parent envelope and therefore classified as a Class 0/I Young Stellar Object (YSO). The embedded phase, lasting more than $10^{5}$ yr (Shu, Adams \& Lizano 1987; Evans II et al. 2009; Dunham et al. 2015), poses observational limitations as radiation from the protostar is heavily attenuated by the optically thick envelope and reprocessed to far-infrared (IR) through (sub-) mm wavelengths. A common approach to determine the nature of embedded YSOs is to consider their Spectral Energy Distributions (SEDs) and their derived bolometric properties (Myers \& Ladd 1993; Andre, Ward-

\footnotetext{
^E-mail: dstamatellos@uclan.ac.uk
}

Thompson \& Barsony 2000; Dunham \& Vorobyov 2012; Sadavoy et al. 2014).

As a protostar accretes gas, either mediated through its protostellar disc or directly from the surrounding envelope, gravitational potential energy is converted to heat on the surface of the protostar and is radiated away. Early theoretical models (Shu 1977; Shu et al. 1987) assumed a constant growth of mass for the protostar. For a solar-mass star, an accretion rate of $\dot{M} \sim 10^{-5} \mathrm{M}_{\odot} \mathrm{yr}^{-1}$ results in a mean luminosity of $\sim 50 \mathrm{~L}_{\odot}$ over the course of its protostellar lifetime (Stahler, Shu \& Taam 1980a,b). However, observations show that typical YSO luminosities are not that high. The earliest work indicating such a disparity was presented by Kenyon et al. (1990), who found that the mean bolometric luminosity of embedded protostars is $\sim 1 \mathrm{~L}_{\odot}$. More recent surveys with higher numbers of observed YSOs confirm this issue, referred to as the luminosity problem (Enoch et al. 2009; Evans et al. 2009; Dunham et al. 2015). One possible solution to this conundrum is that the 
protostellar mass growth happens episodically, in short but intense events (Hartmann \& Kenyon 1985; Kenyon et al. 1990; Hartmann \& Kenyon 1996; Dunham \& Vorobyov 2012; Hartmann, Herczeg \& Calvet 2016). Observations of young stars, like the FUOr-type (after the archetype FU Orionis; Herbig 1966, 1977) and the EXOr-type (after the archetype EX Lupus; Herbig 1989, 2008) objects, provide evidence for such behaviour.

FUOrs are characterized by brightening of up to 5 mag (Herbig 1966, 1977), and have outburst bolometric luminosities of $\sim$ few $100 \mathrm{~L}_{\odot}$ (Audard et al. 2014). The duration of FUOr-type outbursts may last up to decades - e.g. the archetype FU Orionis has only undergone a dimming of 1 mag since its observed 5 mag brightening in 1936 (Herbig 1966, 1977; Kenyon et al. 2000). The periodicity between episodic accretion events has not yet been constrained due to the long dimming duration of currently observed FUOrs, and the limited time of the observations. Suggested driving mechanisms for FUOr-type outbursts include external triggers such as binary-disc interactions (Reipurth \& Aspin 2004) and stellar fly-bys (Pfalzner 2008; Pfalzner, Tackenberg \& Steinhausen 2008). Additionally, instabilities, taking place within the protostellar disc, have also been widely proposed, e.g. thermal instabilities (Bell et al. 1995; Clarke \& Syer 1996; Lodato \& Clarke 2004), gravitational instabilities (Vorobyov \& Basu 2005, 2006, 2010), and a combination of gravitational instabilities and the magneto-rotational instability (Armitage, Livio \& Pringle 2001; Zhu, Hartmann \& Gammie 2009a; Zhu et al. 2009b, 2010a; Zhu, Hartmann \& Gammie 2010b; Stamatellos, Whitworth \& Hubber 2011; Mercer \& Stamatellos 2017).

EXOrs are less intense, episodically accreting protostars. These objects are characterized by bolometric luminosities of $1-2 \mathrm{~L}_{\odot}$, with outburst luminosities rising to tens of $\mathrm{L}_{\odot}$ (Lorenzetti et al. 2006; Sipos et al. 2009; Audard et al. 2010; Sipos et al. 2009; Aspin et al. 2010). EXOrs' periodicity is on the order of weeks to months (Coffey, Downes \& Ray 2004; Audard et al. 2010), with duration between accretion events being on the order of 1-2 yr (Herbig 2008).

If FUOr-type outbursts are due to disc gravitational instabilities, one should expect a tendency for FUOr eruptive events to be common in the embedded phase. There is growing evidence that some of the FUOrs are embedded, as shown by continuum excess at $>100 \mu \mathrm{m}$ (Millan-Gabet et al. 2006). Recent observations have classified some FUOrs as Class 0/I objects (Caratti o Garatti et al. 2011; Kóspál et al. 2011; Safron et al. 2015). Additional support for gravitationally unstable discs triggering FUOrs is provided by the observations of Liu et al. (2016), who showed that there are disc asymmetries, possibly spiral arms, in the NIR, suggesting strong disc self-gravity. An embedded FU Ori-type object is difficult to observe directly as radiation is absorbed very close to the protostar and re-emitted at longer wavelengths (e.g. OO Serpentis, Kóspál et al. 2007; NGC 6334l:MM1 Hunter et al. 2017; HOPS 383, Safron et al. 2015; EC 53, Yoo et al. 2017).

Johnstone et al. (2013) suggested that variations in the far IR through (sub-)mm emission from protostars might be observed as a proxy for episodic accretion, although they found that there may be a delay in the (sub-)mm response of weeks to months after the outburst has started. Monitoring at (sub-)mm wavelengths could help determine the characteristics of the outburst (e.g. rise time, magnitude). Therefore, long-term monitoring of star formation regions at (sub-)mm wavelengths is required. To this end, the James Clerk Maxwell Telescope (JCMT) TRANSIENT Survey is currently undertaking monitoring of eight star-forming regions within $500 \mathrm{pc}$ (Herczeg et al. 2017). The primary aim of this survey is to observe continuum variability, which may relate to episodic accretion events in YSOs. The SCUBA2 camera on the JCMT can provide a reliable measure of relative flux brightness changes to 2-3 per cent for the brightest sources (Mairs et al. 2017). Similarly, ALMA is able to observe variability of a few percent at $1.3 \mathrm{~mm}$ (Francis et al. 2019). Johnstone et al. (2018) summarizes the results of first 18 months of the JCMT Transient Survey, which has uncovered quasi-periodic variability in the YSO EC 53, with an amplitude of 1.4 at $850 \mu \mathrm{m}$ (Yoo et al. 2017), as well as lower amplitude changes from several additional objects over 18 months to $5 \mathrm{yr}$ (Mairs et al. 2018).

Kuffmeier et al. (2018) investigated the flux increase at wavelengths $20-1000 \mu \mathrm{m}$ for heavily embedded YSOs undergoing episodic accretion. Using high-resolution hydrodynamic simulations of star-forming regions, Kuffmeier et al. (2018) modelled the impact of disc instabilities and environment on episodic accretion. They found that late infall of material can result in episodic accretion, either when infall happens onto the disc (promoting disc gravitational instabilities) or directly onto the protostar. For their highest resolution simulation, the most vigorously accreting star undergoes variations in bolometric luminosity by a factor of $\sim 2$. Taking the inclination-mean flux increase between quiescence and outburst phases, they calculate a factor of 5.5, 2.6, 2.0, and 1.2 increase at wavelengths of $25,70,100$, and $1000 \mu \mathrm{m}$, respectively. These flux increases happen on a time-scale of decades to centuries. The authors note that although the continuum flux increases are smaller than observed in classical FUOrs, the variability found in their models is larger than currently observed for non-eruptive young protostars (Rebull et al. 2015; Flaherty et al. 2016; Rigon et al. 2017). The variability of their models is also larger than that found by the TRANSIENT Survey (Mairs et al. 2017; Johnstone et al. 2018; Mairs et al. 2018), which reports secular changes of order 5-10 percent per year from about 10 percent of the protostars bright enough to get good measurements. If these observed sub$\mathrm{mm}$ secular changes continue for time-scales of decades then the magnitude of the flux change will reach a factor of two or more.

Here, we investigate the impact of episodic accretion on the dust continuum emission of a YSO by carrying out radiative transfer modelling of YSOs produced in hydrodynamic simulations of cloud collapse that employ episodic accretion. We aim to determine both the change in the bolometric properties and in the continuum flux response to an episodic accretion event. More specifically our goal is to connect the flux increase at various wavelengths with the actual increase in the luminosity of the embedded protostar during an outburst event. In the follow-up paper (MacFarlane et al., in preparation; hereafter Paper II), we expand on the results of this paper by considering a broader range of protostellar luminosities and structural properties, allowing us to mimic FUOr-type events.

This paper is structured as follows. In Section 2 we describe the hydrodynamic simulations of forming YSOs in collapsing molecular clouds. In Section 3 we describe the radiative transfer code that we use to produce SEDs. We then evaluate YSO SEDs, adopting protostellar properties from the hydrodynamic simulations (Section 4) with or without the influence of heating from an interstellar radiation field. Finally, in Section 5 we summarize our results.

\section{RADIATION HYDRODYNAMIC SIMULATIONS}

We use the Smoothed Particle Hydrodynamics (SPH) code SEREN (Hubber et al. 2011a,b) to follow the evolution of a pre-stellar core with a total mass of $5.4 \mathrm{M}_{\odot}$ (Stamatellos, Whitworth \& Hubber 
2012) that collapses and forms a protostar. If we assume a typical star formation efficiency of $\sim 20$ per cent (e.g. André et al. 2014), then this cloud will end up forming roughly a solar-mass star. The initial density of the pre-stellar core follows a Plummer-like profile

$\rho(r)=\frac{\rho_{\mathrm{C}}}{\left[1+\left(r / R_{\mathrm{C}}\right)^{2}\right]^{2}}$,

where $\rho_{\mathrm{C}}=3 \times 10^{-18} \mathrm{gcm}^{-3}$ is the central density, and $R_{\mathrm{C}}=$ $5000 \mathrm{au}$ is the radius at which the density flattens to $\rho_{\mathrm{C}}$. The radial extent of the core is $R_{\mathrm{CORE}}=50000 \mathrm{au}$, although most of the mass of the core lies within the inner $10000 \mathrm{au}$. As per observed values of typical pre-stellar cores (e.g. André et al. 2014), the initial gas temperature is set to $T=10 \mathrm{~K}$. We impose a random, divergence-free, turbulent velocity field (Bate 2009), with power spectrum $P_{k} d k \propto k^{-4} d k$, to match the scaling laws in molecular clouds (Larson 1981; Burkert \& Bodenheimer 2000), and amplitude such that $\alpha_{\text {TURB }} \equiv U_{\text {TURB }} /\left|U_{\text {GRAV }}\right|=0.3$ (Jijina, Myers \& Adams 1999). The simulation uses $10^{6} \mathrm{SPH}$ particles, such that the mass of each particle is $m_{\mathrm{SPH}}=5.4 \times 10^{-6} \mathrm{M}_{\odot}$. Setting the number of SPH neighbours to $N_{\mathrm{NEIGH}}=50$, the minimum resolvable mass is $M_{\text {min }} \sim m_{\mathrm{SPH}} N_{\mathrm{NEIGH}} \sim 3 \times 10^{-4} \mathrm{M}_{\odot}$. The code uses an artificial viscosity to treat shocks, which is time-dependent in order to avoid unwanted viscosity away from shocks (Morris \& Monaghan 1997).

The radiative transfer method of Stamatellos et al. (2007) is employed, whereby the SPH particle density, temperature, and gravitational potential are used to estimate the optical depth through which the gas cools and heats (see Stamatellos et al. 2007). This is then used to solve the energy equation for every time-step in the simulation. A pseudo-background radiation field is imposed onto the computational domain that ensures that the gas cannot radiatively cool below a certain temperature,

$T_{\mathrm{BGR}}(r)=\left[(10 \mathrm{~K})^{4}+\frac{L_{*}}{16 \pi \sigma_{\mathrm{SB}} r^{2}}\right]^{1 / 4}$,

where $L_{*}$ is the protostellar luminosity, $r$ is the radial distance from the protostar, and $\sigma_{\mathrm{SB}}$ is the Stefan-Boltzmann constant. The first term on the right hand side of the above equation accounts for a background radiation field with a temperature of $10 \mathrm{~K}$, and the second term accounts for heating from the young protostar (see Stamatellos et al. 2011, 2012, for details). The luminosity of the protostar is due to the intrinsic protostellar emission and (mainly) due to accretion of gas onto its surface. This luminosity is parametrized by the protostellar mass $M_{*}$, radius $R_{*}$, and accretion rate $\dot{M}_{*}$, such that

$L_{*}=\left(\frac{M_{*}}{M_{\odot}}\right)^{3} L_{\odot}+L_{\mathrm{acc}}$,

where

$L_{\mathrm{acc}}=f 10.5\left(\frac{M_{*}}{M_{\odot}}\right)\left(\frac{\dot{M}_{*}}{10^{-6} \mathrm{M}_{\odot} \mathrm{yr}^{-1}}\right)\left(\frac{R_{*}}{3 R_{\odot}}\right)^{-1} L_{\odot}$,

and $f=0.75$ is an efficiency parameter which determines the fraction of the gravitational potential energy radiated away by the protostar, rather than convected into the protostar or being used to drive jest and/or winds (Pudritz et al. 2007; Offner et al. 2009; Hartmann, Zhu \& Calvet 2011). The radius of the protostar is set to $R_{*}=3 R_{\odot}$. The effective temperature of the protostar at each computational time-step is computed as

$T_{*}=\left(\frac{L_{*}}{4 \pi R_{*}^{2} \sigma_{\mathrm{SB}}}\right)^{1 / 4}$.

For the simulation analysed in this work, the collapse of the molecular cloud leads to the formation of a protostar at $\sim 79 \mathrm{kyr}$ since the start of the collapse. We assume that a protostar has formed when the density at the centre of the cloud has increased to $10^{-9} \mathrm{~g} \mathrm{~cm}^{-3}$. The protostar is surrounded by a protostellar disc and an evolving infalling envelope. Accretion of gas onto the protostar occurs episodically (see Stamatellos et al. 2011, 2012), resulting in episodic radiative feedback. Fig. 1 presents four characteristic snapshots from the simulation (two before and two during an outburst). The times of these snapshots are marked in Fig. 2, which presents the evolution of the protostellar accretion rate, the protostellar mass, and the protostellar luminosity.

The episodic accretion in this simulation is due to a combination of gravitational instabilities (GI) operating in the outer disc, driving material towards the protostar, and the magneto-rotational instability (MRI) operating in the inner disc region around the young protostar when the conditions are appropriate for a sufficient ionization fraction (Armitage et al. 2001; Zhu et al. 2009a,b, 2010a; Zhu et al. 2010b; Stamatellos et al. 2011; Mercer \& Stamatellos 2017).

As the disc increases in mass by accreting infalling gas from the envelope it becomes gravitationally unstable, as suggested by the formation of the spiral structures seen in the quiescent phase snapshots in the left column of Fig. 1. Thus, angular momentum is redistributed outwards efficiently resulting in inward gas flow. The inner disc is hotter and gravitationally stable such that there is no mechanism available to redistribute angular momentum, and material accumulates around the protostar $(\sim 1 \mathrm{au})$. As more gas flows into this region, compressive, viscous, and protostellar heating raise the local temperature further. Once a threshold temperature of $1400 \mathrm{~K}$ is reached, this region is sufficiently ionized for the MRI to be activated (Zhu et al. 2009a,b, 2010a; Zhu et al. 2010b). The MRI increases the viscosity (the effective viscosity parameter becomes $\alpha_{\text {MRI }}=0.1$; Shakura \& Sunyaev 1973), providing a way to redistribute angular momentum and resulting in rapid gas accretion onto the protostar. During the outburst phase, the accretion luminosity increases dramatically $\left(\sim 10^{3} \mathrm{~L}_{\odot}\right)$, heating the disc and disrupting the spiral structure (outbursting phase snapshots in the left column of Fig. 1; see also MacFarlane \& Stamatellos 2017).

Once the gas from the inner region has been accreted, the protostar returns to the quiescent phase. The process of quiescent to outbursting phase repeats multiple times during the evolution of the YSO (Stamatellos et al. 2012). Outbursts happen every $\sim 1000$ yr initially, whereas at later stages (but still during the Class O/I stage) every $\sim 10000 \mathrm{yr}$ (see Stamatellos et al. 2011, 2012). This is similar to what is inferred from observations (e.g. Scholz, Froebrich \& Wood 2013; Hillenbrand \& Findeisen 2015; Fischer, Safron \& Megeath 2019; see also Peña, Naylor \& Morrell 2019, for longer outburst time-scale estimates for older, Class I/II, YSOs). It is assumed that in the quiescent phase gas accretes onto the protostar at a rate of $\dot{M}=10^{-6} \mathrm{M}_{\odot} \mathrm{yr}^{-1}$ (this is a free parameter in our calculation) and the protostellar luminosity is then on the order of $\sim 1 \mathrm{~L}_{\odot}$. As a comparison, at the same time the accretion rate onto the outer disc is on the order of $\sim 10^{-5}-10^{-4} \mathrm{M}_{\odot} \mathrm{yr}^{-1}$. During the outburst phase the accretion onto the protostar is significantly enhanced. 

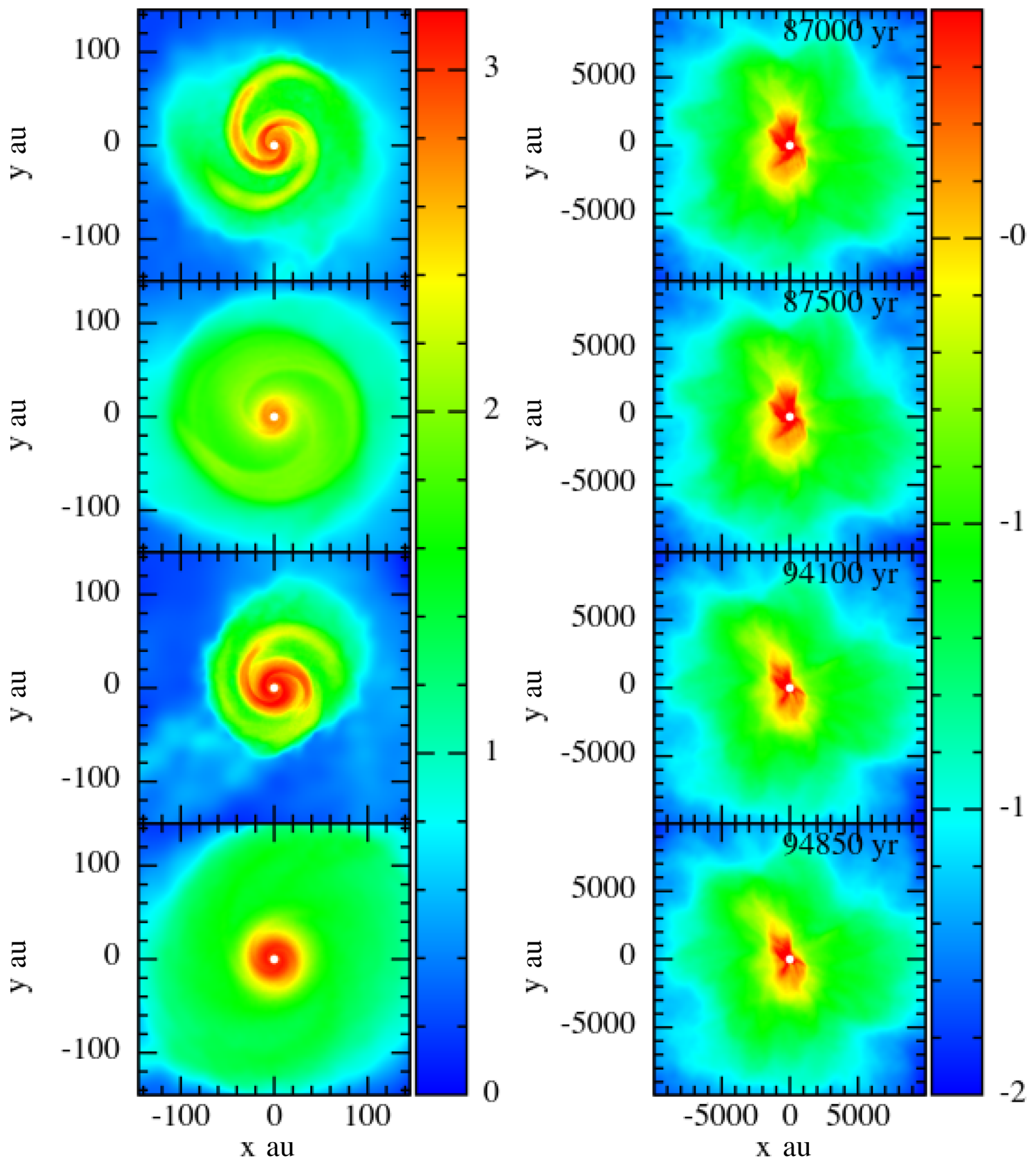

Figure 1. Column density maps (logarithmically scaled; $\mathrm{gcm}^{-2}$ ) of four snapshots during the collapse of a pre-stellar core forming a young protostar and disc. The left column shows the small-scale structure (disc) and the right column shows the large-scale structure (envelope) surrounding the YSO. The snapshots corresponds to the quiescent and outbursting phases of two events, termed as E1 and E2 (see also Fig. 2). From top to bottom, the snapshots are the following: E1-quiescent phase, E1-outbursting phase, E2-quiescent phase, E2-outbursting phase. The effect of the outburst on the disc is dramatic (see the left column). On the other hand, the large-scale structure of the YSO does not significantly change during the outburst (see the right column).

This model is different than the models of Vorobyov \& Basu (2005, 2006, 2010). In their models the GIs alone are responsible for the observed outbursts: GIs create clumps in the disc of a young stellar object that eventually fall onto the protostar, resulting in outbursts (Dunham et al. 2013). Nevertheless, both models have the same outcome: increased accretion rate onto the young protostar. We expect that the SED versus accretion luminosity relationship found in this paper should hold in both cases. 

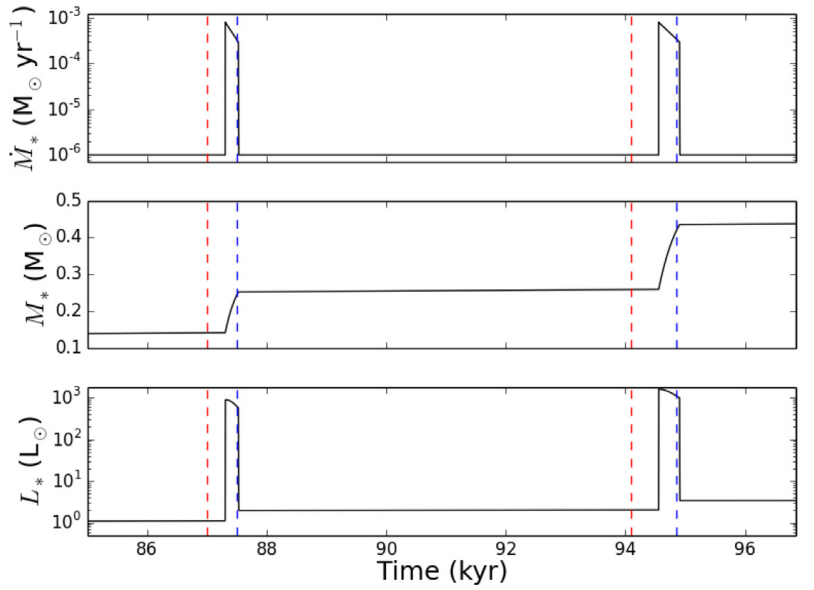

Figure 2. Evolution of the protostellar accretion rate $\dot{M}_{*}$ (top), mass $M_{*}$ (middle), and luminosity $L_{*}$ (bottom) for the simulation analysed in this paper. Red and blue dashed lines define the times at which quiescent and outbursting snapshots are selected for detailed radiative transfer calculations. Each pair of red and blue dashed lines correspond to the accretion events E1 and E2, respectively.

\section{RADIATIVE TRANSFER MODELLING}

\subsection{Radiative transfer methods}

The radiative transfer employed within the hydrodynamic simulation uses the diffusion approximation method (Stamatellos et al. 2007). Thus, the computed temperatures are not accurate enough to be compared against observations. Hence, we post-process the simulations using polychromatic radiative transfer (RT) to model different snapshots in time.

We employ the 3D radiative transfer code RADMC-3 $\mathrm{D}^{1}$ (Dullemond et al. 2012) that uses the Monte Carlo Radiative Transfer (MCRT) method of Bjorkman \& Wood (2001) to compute the equilibrium temperature for a density distribution given a set of luminosity sources and a wavelength-dependent opacity (see also Stamatellos \& Whitworth 2003; Stamatellos 2003; Stamatellos et al. 2004). The code works by randomly emitting and subsequently propagating photon packets (henceforth simply referred to as photons) from each luminosity source within the computational domain. A photon propagates a distance within the computational domain depending on its randomly sampled optical depth. Once a photon is absorbed, its energy is deposited at that location, and the photon is re-emitted in a random direction. The method uses a modified random walk algorithm (Min et al. 2009; Robitaille 2010) to reduce the computation time required when a photon travels through optically thick regions. Once all photons have propagated through the system, the equilibrium temperature of the system is determined. RADMC-3D then is used to solve the equation of radiative transfer and calculate the wavelength-dependent source function towards an observer location. This method, denoted as Ray-tracing Radiative Transfer (RRT), is used to produce synthetic observations (SEDs and images) of different snapshots of the simulation.

\footnotetext{
${ }^{1}$ http://www.ita.uni-heidelberg.de/ dullemond/software/radmc-3d/ $\quad$ (Last
} accessed: 07/07/2018)

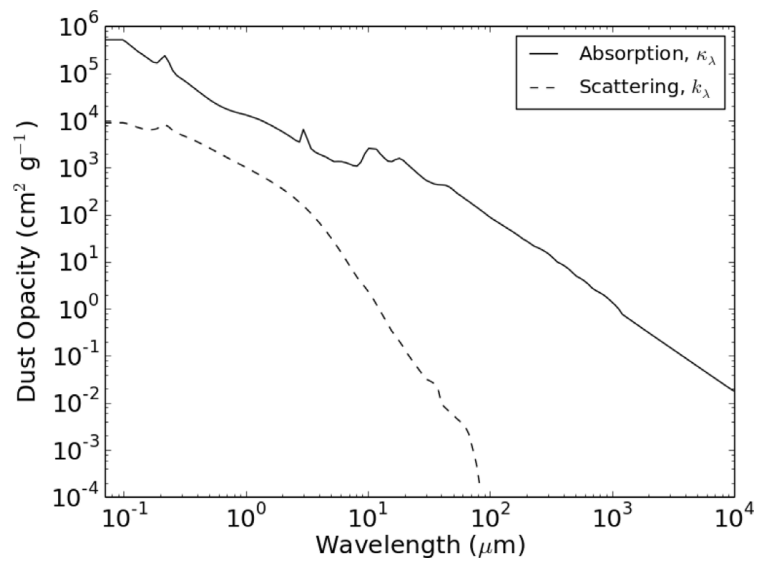

Figure 3. Ossenkopf \& Henning (1994; OH5) dust absorption (solid line) and scattering (dashed line) opacities.

\subsection{Construction of the radiative transfer grid}

RADMC-3D adopts a grid for numerical computations. As such, the SPH density distribution, represented by a large number of SPH particles, must be translated to a grid (Stamatellos \& Whitworth 2005). To this end, we adopt an adaptive-mesh refinement method. Initially, we construct a grid cell encapsulating the entire computational domain. This cell is then divided into equal-volume octants. Each of these sub-cells is then divided into octants and the process continues until each cell contains a user-specified number of SPH particles or reaches the maximum refinement level (defined by the user). The algorithm adopted here follows that in the RT code HYPERION (Robitaille 2011). For the following analyses, the recursive conditions to build the grid are that each grid volume hosts $\leq 10$ SPH particles, or a maximum refinement level of 20 has been reached. These two criteria ensure adequate resolution in the most optically thick regions - namely the protostellar disc and the inner envelope. The computed gas density distribution is then converted to a dust density, adopting a nominal dust-to-gas ratio of 1:100.

Near the protostar the temperature is high enough for dust to sublimate (Lenzuni, Gail \& Henning 1995; Duschl, Gail \& Tscharnuter 1996). We calculate the dust destruction radius, $R_{\mathrm{DUST}}$, around the protostar as

$R_{\text {DUST }}=\frac{R_{*}}{2}\left(\frac{T_{*}}{T_{\text {destr }}}\right)^{\left(4+\beta_{\mathrm{d}}\right) / 2}$,

by assuming that the dust heated by the protostar is in thermal equilibrium. $T_{*}$ is computed using equation $3, \beta_{\mathrm{d}}=3$ is the dust spectral opacity index $\left(1 \lesssim \beta_{\mathrm{d}} \lesssim 3\right.$; see e.g. Natta \& Testi 2004), and $T_{\text {destr }}=1200 \mathrm{~K}$ is the assumed dust destruction temperature. All cells interior to $R_{\text {DUST }}$ are then set to have zero density.

\subsection{Dust opacities}

We use the opacities of Ossenkopf \& Henning (1994; OH5), for dust grains of a standard MRN mixture (Mathis, Rumpl \& Nordsieck 1977; 53 per cent silicate, 47 per cent graphite) with thin ice mantles at a density of $10^{6} \mathrm{~cm}^{-3}$ (see Fig. 3). The wavelength range for this opacity table is extended to values of $\lambda<1 \mu \mathrm{m}$ by adopting the optical constants of Draine \& Lee (1984) for the MRN mixture, computing the absorption and scattering opacities, and rescaling them to match the $\mathrm{OH} 5$ values at $1 \mu \mathrm{m}$. 


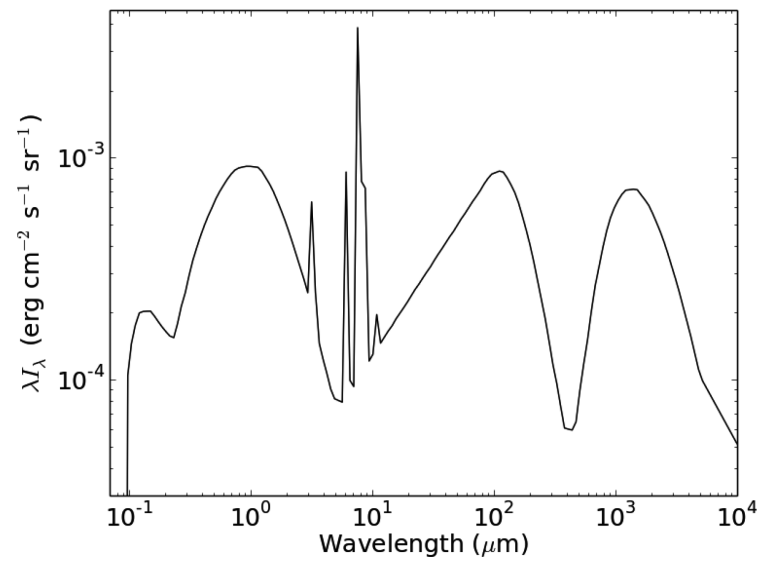

Figure 4. Intensity distribution of the André et al. (2003) interstellar radiation field adopted during the radiative transfer modelling.

\subsection{Radiation sources}

We consider two sources of radiation: (i) the young protostar, and (ii) the interstellar radiation field (ISRF).

In the RT simulations presented in this paper we adopt the protostellar luminosity provided by the hydrodynamic simulation (in Paper II, we treat the protostellar luminosity as a free parameter so that we can explore a larger parameter space). The emission from the protostar is assumed to follow a blackbody profile at the protostellar temperature. The protostellar radiation is absorbed and re-emitted very close to the protostar; therefore, the blackbody assumption does not affect the results presented in this paper.

The ISRF (taken into account for a selected set of simulations) considers contributions to the radiation field from the cosmic microwave background, the galactic dust emission, and irradiation from background stars. We adopt the ISRF of André et al. (2003), which is a modified Black (1994) ISRF, accounting for emission from poly-aromatic hydrocarbons (Fig. 4). It is assumed that the ISRF isotropically heats from the outside the whole cloud or computational domain (i.e. at $\sim 50000 \mathrm{au}$ ), therefore the radiation is somewhat attenuated by the envelope material before it reaches the YSO that we model (i.e. the inner 10000 au of the cloud).

\section{RADIATIVE TRANSFER MODELLING OF YSOS}

We focus on two episodic accretion or outburst events in the following modelling and analyses - labelled E1 and E2, respectively (see Fig. 2). E1 and E2 are both characterized by protostellar luminosity increases of a factor of $\sim 550$, with outbursting phase durations of $\sim 200$ and $\sim 400 \mathrm{yr}$, respectively. A simulation snapshot before the outburst phase, and a snapshot during the outburst phase are selected for each of events E1 and E2. These are denoted by the red (quiescent - at 87.00 and $94.10 \mathrm{kyr}$ ) and blue (outbursting - at 87.50 and $94.85 \mathrm{kyr}$ ) dashed lines of Fig. 2, respectively. These snapshots correspond to the column density maps presented in Fig. 1. The outburst snapshots for both events were chosen near the end of each outburst so that the region around the protostar has time to adjust itself to the additional heating provided during the outburst (MacFarlane \& Stamatellos 2017).

MCRT is carried out on each of these snapshots. We examine two models, (i) including and (ii) excluding the contribution from the ISRF. For the MCRT computations $10^{6}$ photon packets are used to represent the radiation from the protostar and $10^{7}$ photon packets for the ISRF. These values ensure that the statistical noise when calculating the equilibrium temperature is similar for both types of luminosity sources.

\subsection{Temperature distributions}

The $x-y$ temperature maps (taken at $z=0$ au, i.e. at the disc mid-plane) for event E1 are presented in Fig. 5. Left- and righthand panels represent the temperature profiles for quiescent and outbursting snapshots, respectively. The top or bottom panels represent MCRT computations with or without heating contributions from the ISRF.

For MCRT models that include ISRF heating, the temperature reaches a minimum within the envelope, at a radius determined by the relative strength of the ISRF with respect to the protostellar luminosity. The radius of the temperature minima for the quiescent phase snapshot we present in Fig. 5 (top left) is $\sim 12000$ au ( $\sim 5 \mathrm{~K})$, whereas for the outburst phase (top right) it is $\sim 23000$ au ( $\sim 15 \mathrm{~K}$ ).

For MCRT models where the ISRF heating is neglected, the temperature decreases monotonically with distance from the central protostar. The temperature minima, therefore, occur at the outer edge of the envelope ( $\sim 50000 \mathrm{au})$ : the minimum temperature is $\sim 5 \mathrm{~K}$ for the quiescent (Fig. 5, bottom left) and $\sim 15 \mathrm{~K}$ for the outbursting model (Fig. 5, bottom right).

\subsection{Spectral energy distributions}

We use the calculated temperature distributions to produce SEDs for each snapshot. For these calculations in RADMC-3D, we take into account only the radiation that comes from the region $R \leq 10000$ au around the protostar. We therefore assume that any background emission (i.e. any emission outside 10000 au) is filtered out either directly or removed by considering external annuli and subtracting to only get the flux associated with the source. Our observed region is therefore roughly the typical size of a Class 0 object as mapped in the sub-mm (e.g. at $850 \mu \mathrm{m}$ by JCMT). Considering the distances of nearby star-forming regions where such objects are observed $(150-500 \mathrm{pc})$ and the resolution of the JCMT at $850 \mu \mathrm{m}(14 . " 6)$, then the spatial resolution is $\sim 2200-7300$ au, which provides a lower limit on the integration area. In Paper II we also discuss integration areas of 1000 au that can be probed by higher resolution observations, e.g. by ALMA. We adopt a distance to the source of $d=140 \mathrm{pc}$ and an inclination of $i=0^{\circ}$. We define $i=0^{\circ}$ to be the orientation such that the protostellar disc of the YSO is face-on.

We present SEDs for all snapshots and luminosity source combinations in Fig. 6. Top and bottom panels represent SEDs for events $\mathrm{E} 1$ and $\mathrm{E} 2$, with red or blue lines indicating the emission from quiescent or outbursting phase. Dashed or solid lines represent temperature distributions computed with or without ISRF contributions. On the right column of Fig. 6, we present the ratio between the outburst and the quiescent phase flux over a narrower wavelength range to better illustrate the differences between the models with and without ISRF.

\subsection{Flux increases in episodically accreting protostars}

The ratio of outbursting to quiescent flux at long wavelengths serves as a proxy for the change in protostellar luminosity during an accretion event. In Table 1 we present the outbursting and 

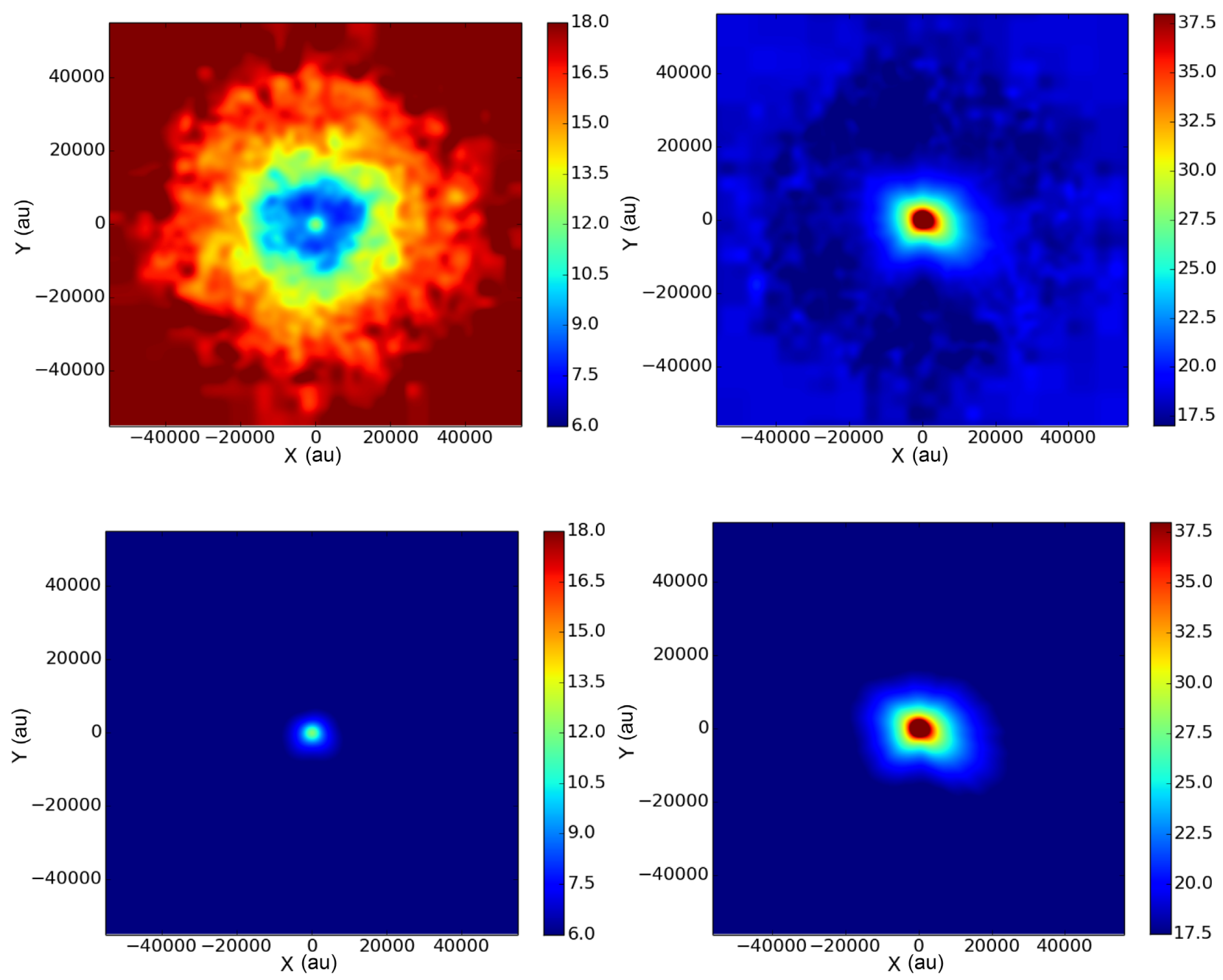

Figure 5. Temperature distributions on the $x-y$ plane ( $z=0 \mathrm{au}$; in Kelvin), for radiative transfer models of event E1. Left- and right-hand panels represent quiescent and outbursting phases, respectively, while top and bottom panels represent MCRT calculations with and without ISRF contributions, respectively.

quiscent luminosities for each event. We also present the flux ratios between outbursting and quiescent snapshots (see SEDs of Fig. 6). We compute ratios at wavelengths corresponding to continuum bands of the Herschel Space Observatory, JCMT, and the Atacama Large (Sub-) Millimetre Array (ALMA; 70, 250,350,450, 850, and $1300 \mu \mathrm{m})$.

In the quiescent phase SEDs, very little flux is emitted at wavelengths shorter than $\lambda<100 \mu \mathrm{m}$. In contrast, the outburst phase SEDs exhibit significant increases in flux due to the enhanced heating of the envelope. This result suggests that an embedded source that was previously undetectable, may be observed at $\lambda<100 \mu \mathrm{m}$ (e.g. with the upcoming James Webb Space Telescope; Gardner et al. 2006) if the protostar undergoes an outburst.

The magnitude of the flux ratios in our models are much higher than those of EC 53, an embedded Class I YSO. EC 53 underwent an increase in $850 \mu \mathrm{m}$ flux by a factor 1.5 (Yoo et al. 2017), indicative of a factor of $\sim 4$ increase in accretion luminosity. In contrast, the protostar in our hydrodynamic simulation undergoes luminosity increases of order $\sim 550$, leading to flux increases at $850 \mu \mathrm{m}$ an order of magnitude higher than observed in EC 53.

\subsection{Impact of the ISRF on the SEDs}

We see in Fig. 6 that heating from the ISRF results in an increase in flux at mid-IR and longer wavelengths, but only during the quiescent phase of the protostar. In contrast, the outbursting phase SEDs show no discernible change in long wavelength flux when the ISRF heating is included. As noted in Section 4.1, the heating of the envelope is dominated by the ISRF outside $\sim 12000$ au for the quiescent phase and outside $\sim 23000$ au for the outburst phase of event E1. As in both cases the regions in which the ISRF dominates is outside the region over which the SED is computed (10 $000 \mathrm{au})$, only small differences in long wavelength emission are expected. For a larger integration area (say $20000 \mathrm{au}$ ) more significant (but still rather small) differences are expected during the quiescent phase.

Investigating this further, in Fig. 7 we present $\lambda F_{\lambda}$ as a function of the size of the region around the protostar from which we calculate the flux for event E1. Values are computed at wavelengths of 350 and $1300 \mu \mathrm{m}$, including radiation from within an increasing radius in the models that include heating from the ISRF. In the quiescent snapshot (solid lines), there is a noticeable drop in longwavelength emission with decreasing radius. This is because the 

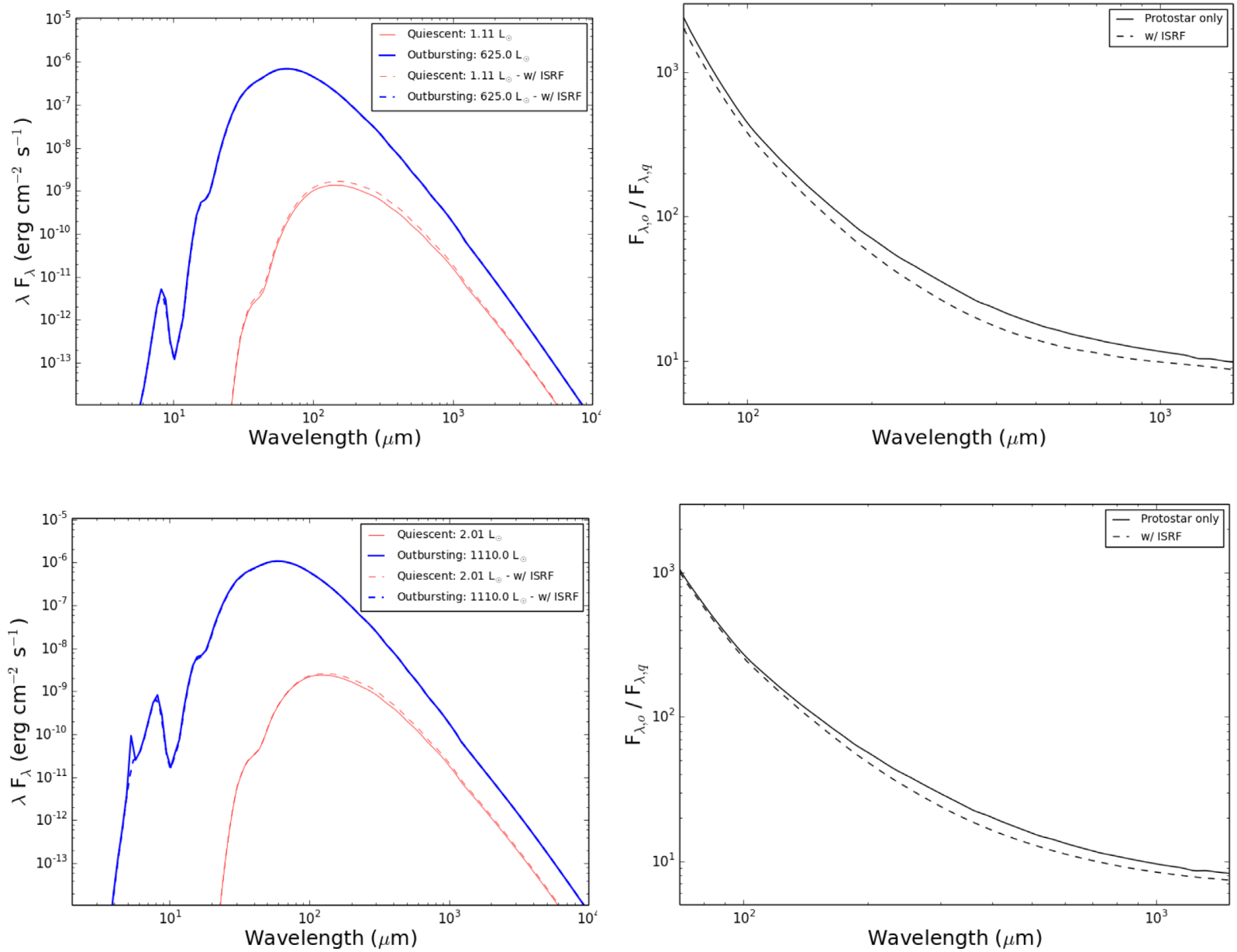

Figure 6. SEDs (left) and the ratios of outbursting-to-quiescent flux (right), with and without heating from the ISRF, for event E1 (top) and E2 (bottom). On the left graphs, red and blue lines represent SEDs for quiescent and outbursting phase snapshots, respectively. Solid or dashed lines correspond to RT models without or with the ISRF (as indicated on each graph).

Table 1. Ratio of fluxes at various wavelengths (outbursting flux. $F_{\lambda, \mathrm{o}}$, divided by quiescent flux, $F_{\lambda, \mathrm{q}}$ ). Ratios are calculated for two accretion events (E1, E2) using the source luminosities adopted in the MCRT models (with or without ISRF). $M_{*}$ is the mass of the protostar, $L^{*}$ is its luminosity (quiescent and outbursting), and $L_{\mathrm{o}}^{*} / L_{\mathrm{q}}^{*}$ is the ratio of outbursting to quiescent luminosity for each event.

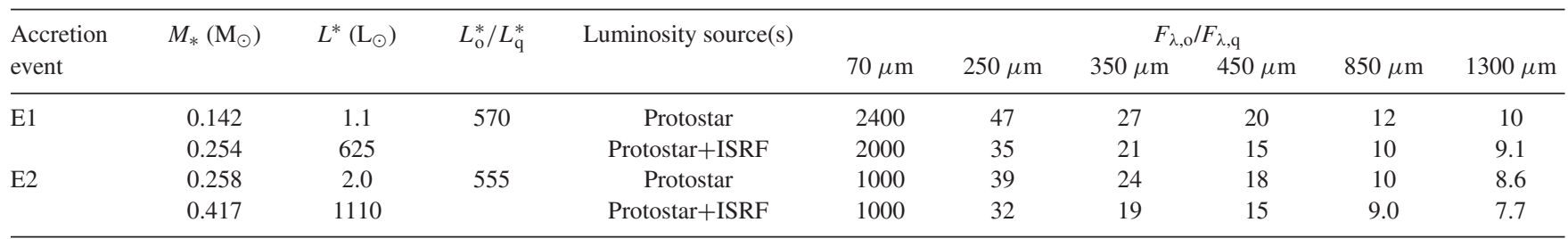

flux from the outer envelope, which is heated by the ISRF, is not included. On the other hand, in the outbursting phase heating of the envelope is due to primarily the protostellar luminosity, and the ISRF contribution is minimal. As a result, there is no significant change in long wavelength emission with decreasing distance from the protostar. This confirms that the relative strengths of the luminosities from protostar and the ISRF determine whether significant long wavelength flux increases are observed, when heating from an ISRF is present.
In the case of strong heating from the ISRF and a relatively small protostellar luminosity increase (as in example, EXOr-type objects), the above effect could lead to just a slight increase in the radiation at long wavelengths if the flux is calculated over a large integration area (i.e. due to low-angular resolution). To mitigate this issue, ideally one should conduct high-resolution observations of the central regions of the YSO (e.g. $\lesssim 10,000-20,000 \mathrm{au}$ ) ensuring that radiation is received from regions where heating is predominantly due to protostellar radiation and not from the ISRF. 


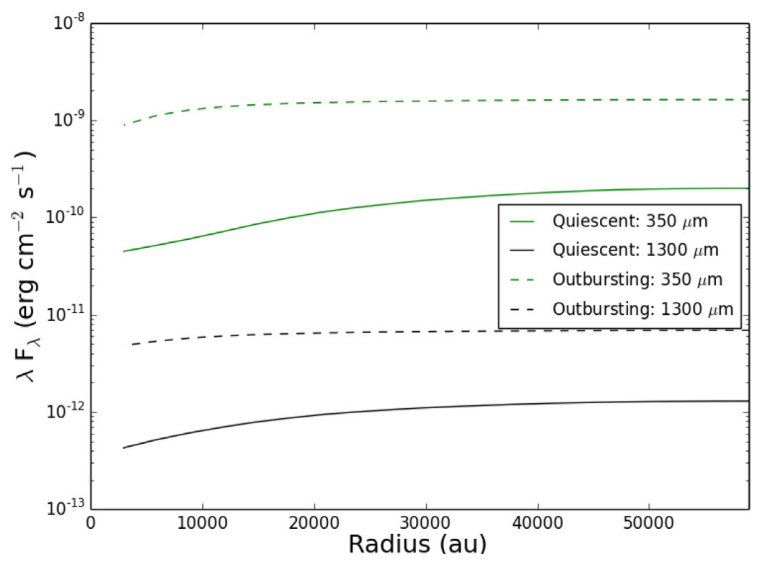

Figure 7. $\lambda F_{\lambda}$ at $350 \mu \mathrm{m}$ (green) and $1300 \mu \mathrm{m}$ (black) for E1 event snapshots, as a function of radius within which radiation is included. Solid lines represent the quiescent phase of event E1, with the outbursting phase snapshot represented by dashed lines. RT calculations for both cases are carried out with both protostellar and ISRF heating.

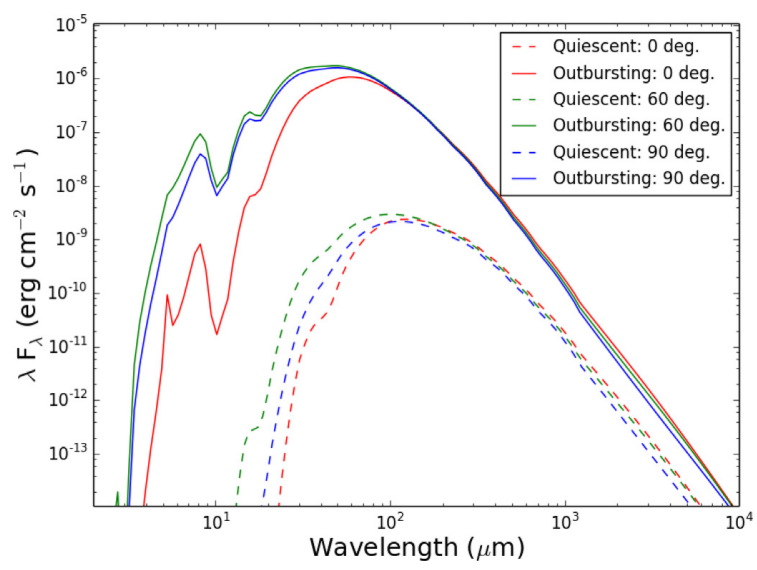

Figure 8. SEDs for event E2 (computed with both the protostellar and ISRF heating), computed at inclinations of $0^{\circ}$ (red), $60^{\circ}$ (green), and $90^{\circ}$ (blue). Quiescent phase SEDs are represented by dashed lines, while solid lines represent the outbursting phase.

\subsection{The effect of inclination on the SEDs}

The observed SEDs of YSOs depend on their orientation. In Fig. 8 we present SEDs for event E2 (with ISRF heating), at inclinations of $0^{\circ}, 60^{\circ}$, and $90^{\circ}$. An inclination of $i=0^{\circ}$ denotes observing onto the $x-y$ plane (disc face-on), whereas an inclination of $i=$ $90^{\circ}$ denotes observing onto the $x-z$ plane (disc edge-on). As mentioned earlier (see Fig. 1), the simulated pre-stellar collapsing cloud evolves to a highly asymmetric state. Fig. 8 demonstrates that for both quiescent and outbursting phases, asymmetries in the envelope lead to a non-monotonic relationship between flux and inclination for $\lambda<200 \mu \mathrm{m}$. This is in contrast to models of Class $0 / I$ YSOs with axisymmetric envelopes flattened due to rotation, in which the increase of inclination from $i=0^{\circ}$ to $i=90^{\circ}$ leads to decreasing flux at all wavelengths (e.g. Whitney et al. 2003a,b). At longer wavelengths (e.g. $1.3 \mathrm{~mm}$ ), the flux decreases with increasing inclination in both set of models.

Radiation at $\lambda<200 \mu \mathrm{m}$ is attenuated due to the relatively highdust opacity, so that the radiation received by the observer is from
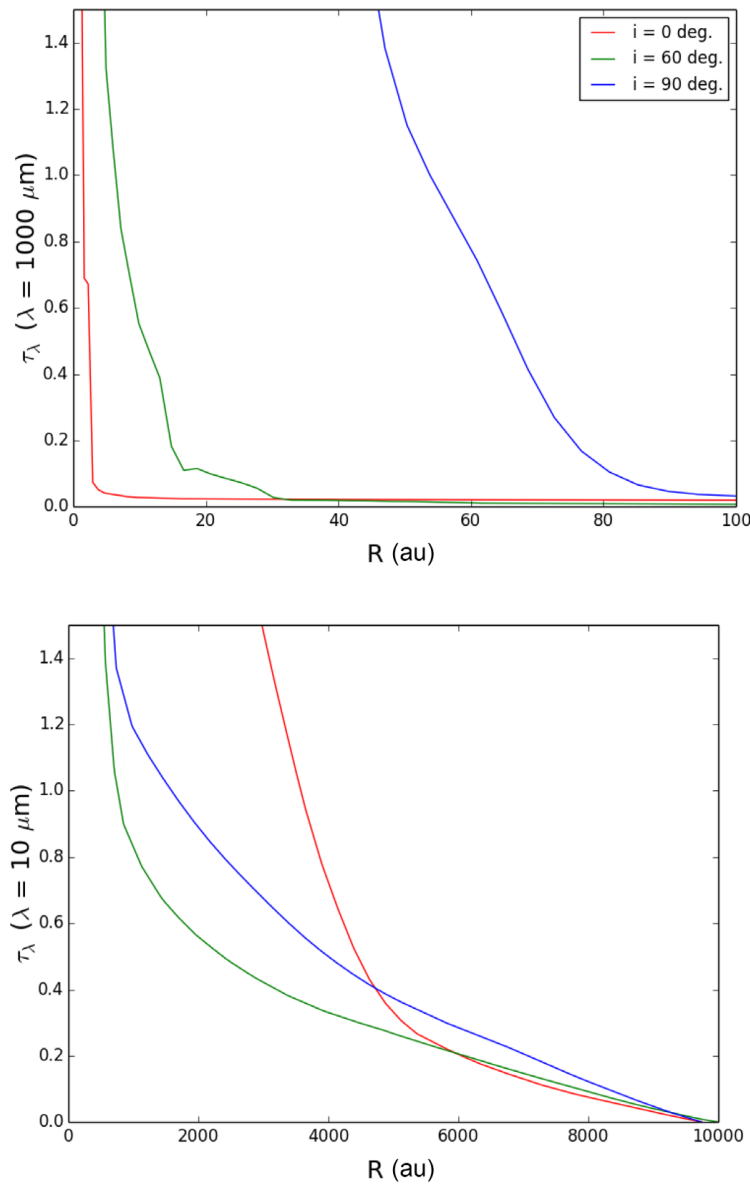

Figure 9. Optical depth from the observer to a specific radius $R$ away from the protostar at different viewing angles for $\lambda=1000 \mu \mathrm{m}$ (top) and $\lambda=10 \mu \mathrm{m}$ (bottom). In each panel, red, green, and blue lines denote an inclination of $0^{\circ}, 60^{\circ}$, and $90^{\circ}$, respectively.

the outer layers of the envelope. The SEDs presented imply that at $60^{\circ}$ the radiation comes from deeper within the envelope than at $0^{\circ}$ or $90^{\circ}$. This radiation comes from the inner, hotter, and more dense envelope at $i=60^{\circ}$, compared to $i=0^{\circ}, i=90^{\circ}$. On the other hand, radiation at $\lambda>200 \mu \mathrm{m}$, comes from almost the entire YSO (i.e. even from the inner parts of the envelope), and it decreases slightly with increasing inclination.

Investigating further, in Fig. 9 we present radial optical depth profiles, for $\lambda=10 \mu \mathrm{m}$ and $\lambda=1000 \mu \mathrm{m}$. This calculation is carried out for the quiescent phase snapshot of E2 (the SEDs of which are presented in Fig. 8), calculating the optical depth from the observer toward the central protostar. The optical depth, $\tau_{\lambda}(R)$, is calculated by numerically integrating the density from $R_{\mathrm{OUT}}=$ $10000 \mathrm{au}$, i.e. at the radial range over which the SEDs of Fig. 8 are computed to a given radius $R$, i.e.

$\tau_{\lambda}(R)=-\kappa_{\lambda} \int_{R_{\mathrm{OUT}}}^{R} \rho(r) \mathrm{d} r$,

where $\kappa_{\lambda}$ is the dust opacity. As the outer density of the cloud drops approximately as $r^{-4}$ (see equation 2), the contribution to the optical depth from dust outside 10000 au at these long wavelengths is minimal. The top panel of Fig. 9 shows that at $\lambda=1000 \mu \mathrm{m}$ radiation comes progressively from deeper within the envelope as the inclination increases (as indicated by the radius at which $\tau_{\lambda} \sim$ 
Table 2. The bolometric luminosity $\left(L_{\mathrm{BOL}}\right)$, bolometric temperature ( $\left.T_{\mathrm{BOL}}\right)$, and sub-mm to bolometric luminosity ratio $\left(L_{\mathrm{sub}-\mathrm{mm}} / L_{\mathrm{BOL}}\right)$ for events E1 and E2 (before and during an outburst) for an inclination of $i=0^{\circ}$.

\begin{tabular}{lcccc}
\hline $\begin{array}{l}\text { Accretion } \\
\text { event }\end{array}$ & $\begin{array}{c}\text { Luminosity } \\
\text { source(s) }\end{array}$ & $\begin{array}{c}L_{\mathrm{BOL}} \\
\left(\mathrm{L}_{\odot}\right)\end{array}$ & $\begin{array}{c}T_{\mathrm{BOL}} \\
(\mathrm{K})\end{array}$ & $\begin{array}{c}\text { L-ratio } \\
(\%)\end{array}$ \\
\hline E1-Quiescent & $\begin{array}{c}\text { Protostar } \\
\text { E1-Outbursting }\end{array}$ & 1.1 & 25 & 8.9 \\
& Protostar+ISRF & 1.3 & 25 & 9.2 \\
E2-Quiescent & Protostar & 500 & 59 & 0.38 \\
& Protostar+ISRF & 500 & 58 & 0.38 \\
E2-Outbursting & Protostar & 1.9 & 29 & 6.2 \\
& Protostar+ISRF & 2.1 & 28 & 6.7 \\
& Protostar & 790 & 66 & 0.26 \\
& Protostar+ISRF & 790 & 65 & 0.26 \\
\hline
\end{tabular}

1). In the case of $\lambda=10 \mu \mathrm{m}$ (left-hand panel of Fig. 9), the radius at which $\tau_{\lambda} \sim 1$ is farther away from the protostar and does not decrease monotonically as the inclination increases. In this case, the radius at which $\tau_{\lambda}=1$ is smaller for $i=60^{\circ}$, and larger for $i=$ $0^{\circ}$, confirming the inclination variation of the SEDs seen in Fig. 8. Therefore, in the non-axisymmetric system that we model, the use of the inclination angle to interpret an SED is not particularly useful.

\subsection{YSO classification}

Each of the model snapshots presented here represent a deeply embedded protostar, i.e. a Class 0 YSO. To classify a YSO, the bolometric temperature and the sub-mm to bolometric luminosity ratio may be used. Sources with a bolometric temperature of $T_{\mathrm{BOL}}<70 \mathrm{~K}$ are classified as Class 0 YSOs (Chen et al. 1995). The bolometric temperature is calculated from the equivalent temperature of a blackbody with the same flux-weighted mean frequency as the YSO. Alternatively, Class 0 YSO sources are identified as those with a ratio of sub-mm to bolometric luminosity, $L_{\text {sub-mm }} / L_{\mathrm{BOL}}>0.5$ per cent (André, Ward-Thompson \& Barsony 1993). The sub-mm luminosity is defined as the integrated flux for wavelengths longer than $350 \mu \mathrm{m}$.

The ratio of the sub-mm to bolometric luminosity and the bolometric temperature are numerically calculated (see Table 2) from the SED,

$\frac{L_{\text {sub-mm }}}{L_{\mathrm{BOL}}}=\frac{\int_{0}^{v(350 \mu \mathrm{m})} F_{v} \mathrm{~d} v}{\int_{0}^{\infty} F_{v} \mathrm{~d} v}$,

and

$T_{\mathrm{BOL}}=1.25 \times 10^{-11} \frac{\int_{0}^{\infty} v F_{v} \mathrm{~d} v}{\int_{0}^{\infty} F_{v} \mathrm{~d} v} \mathrm{~Hz}^{-1} \mathrm{~K}$,

(see Myers \& Ladd 1993).

When using the bolometric temperature, all stages of the evolution of the YSO presented in Table 2 are classified as Class 0 (note, however, that there is also a dependence on inclination; see Paper II). In contrast, when using the ratio of sub-mm to bolometric luminosity, the YSO is classified as a Class 0 object only during its quiescent phase. In the outbursting phase the ratio is $<0.5$ per cent, indicative of later phase YSOs (André et al. 1993). This result is due to a significant fraction of the protostellar radiation in the outbursting phase being emitted at shorter wavelengths, causing the sub-mm to bolometric luminosity ratio to decrease significantly. As the envelope is more massive than the protostar itself, the YSO is still a Class 0 source (André et al. 2003). This implies that using the ratio of sub-mm to bolometric luminosity may lead to an incorrect classification for very luminous FUOrs. Heating from the ISRF has little significant effect on the computed bolometric values and therefore on the classification (see Table 2).

Comparing the $L_{\mathrm{BOL}}$ values we calculate to the observed luminosity distribution of YSOs, we find that the quiescent phase values are comparable to the observed mean values for Class 0/I YSOs ( $\sim 1 \mathrm{~L}_{\odot}$; Evans et al. 2009; Dunham et al. 2013). The bolometric luminosities in the outbursting phase are higher than the typical $L_{\mathrm{BOL}}$ of FU Ori objects $\left(100-300 \mathrm{~L}_{\odot}\right.$; Audard et al. 2014). However, there are a few observations of FU Ori objects with such high luminosities, e.g. Z CMa (400-600L $\odot$; Hartmann \& Kenyon 1996) and V1057 Cyg $\left(250-800 L_{\odot}\right.$; Hartmann \& Kenyon 1996). This indicates that the FU Ori-type objects that we model here represent rather rare events. Quick evolution within the Class 0 phase (e.g. Dunham et al. 2015), and the relatively short duration of outbursts suggests that observations of such luminous YSOs would be rare.

\section{CONCLUSIONS}

In this paper, we presented polychromatic radiative transfer simulations of episodically outbursting YSOs formed in hydrodynamic simulations. The primary aim of this work has been to determine the changes in the continuum flux at various wavelengths and connect these with the luminosity increase of the young protostar. Additionally, we explored the change in the bolometric properties of YSOs due to outburst events.

The radiative transfer models that we presented utilized protostellar properties directly from hydrodynamic simulations that model YSOs undergoing FUOr-type outbursts (see Paper II for a parameter study). SEDs have been computed both in quiescent and outbursting phases for two episodic accretion events. The integrated flux has been computed over the central $R \leq 10000$ au region of each YSO, at various inclinations. The flux increase due to episodic accretion events is more prominent at near-IR (NIR) wavelengths (a factor of up to 50 increase at $250 \mu \mathrm{m}$ ) than at $\mathrm{mm}$ wavelengths (a factor of 10 increase at $1.3 \mathrm{~mm}$ ). The effect of heating from the ISRF may somewhat attenuate the expected flux increase during an episodic accretion event. The reduced flux contrast between quiescent and outbursting phase may be avoided if resolution permits the monitoring of the inner regions of the YSO, where the heating happens primarily due to protostellar radiation (see Paper II).

We have also calculated bolometric luminosities and temperatures before and during outbursts and found that a correct classification as a Class 0 object is generally made when using the bolometric temperature as a proxy for evolutionary phase. However, when using the ratio of sub-mm to bolometric luminosity during outbursts the YSO is often incorrectly classified as Class I, indicating that this criterion is not an optimal indicator of the evolutionary phase for outbursting YSOs.

The work presented in this paper describes the observational characteristics of young Class 0/I protostars undergoing episodic mass accretion and therefore large luminosity outbursts, akin to FU Ori-type objects. In the follow-up paper (Paper II) we consider a range of protostellar luminosity and YSO structure and we find that the flux increases at different wavelengths also depend on the specific density structure and the geometry of the young protostar. We provide diagnostics to infer the luminosity of episodically outbursting embedded protostars using observations at far-IR (FIR) and mm wavelengths. 


\section{ACKNOWLEDGEMENTS}

BM is supported by STFC grant ST/N504014/1. DS is partly supported by STFC grant ST/M000877/1. DJ is supported by the National Research Council Canada and by an NSERC Discovery Grant. GH is supported by general grant 11773002 awarded by the National Science Foundation of China. Simulations were performed using the UCLAN HPC facility and the COSMOS Shared Memory system at DAMTP, University of Cambridge operated on behalf of the STFC DiRAC HPC Facility. This equipment is funded by BIS National E-infrastructure capital grant ST/J005673/1 and STFC grants ST/H008586/1, ST/K00333X/1. SEREN has been developed and maintained by David Hubber, who we thank for his help. Column density maps were generated using the visualization software SPLASH (Price 2007). This work is supported by the JCMT-Transient Team.

\section{REFERENCES}

Andre P., Ward-Thompson D., Barsony M., 1993, ApJ, 406, 122

Andre P., Ward-Thompson D., Barsony M., 2000, in Mannings V., Boss A. P., Russell S. S., eds, Protostars and Planets IV. Univ. Arizona Press, Tucson, p. 59

André P., Bouwman J., Belloche A., Hennebelle P., 2003, in Curry C. L., Fich M., eds, Chemistry as a Diagnostic of Star Formation. NRCPress, Ottawa, p. 127

André P., Di Francesco J., Ward-Thompson D., Inutsuka S.-I., Pudritz R. E., Pineda J. E., 2014, in Beuther H., Klessen R. S., Dullemond C. P., Henning T., eds, Protostars and Planets VI. Univ. Arizona Press, Tucson, p. 27

Armitage P. J., Livio M., Pringle J. E., 2001, MNRAS, 324, 705

Aspin C., Reipurth B., Herczeg G. J., Capak P., 2010, ApJ, 719, L50

Audard M. et al., 2010, A\&A, 511, 26

Audard M. et al., 2014, in Beuther H., Klessen R. S., Dullemond C. P., Henrik T., eds, Protostars and Planets VI. Univ. Arizona Press, Tucson, p. 387

Bate M. R., 2009, MNRAS, 392, 590

Bell K. R., Lin D. N. C., Hartmann L. W., Kenyon S. J., 1995, ApJ, 444, 376

Bjorkman J. E., Wood K., 2001, ApJ, 554, 615

Black J. H., 1994, in Cutri R. M., Latter W. B., eds, ASP Conf. Ser. Vol. 58, The First Symposium on the Infrared Cirrus and Diffuse Interstellar Clouds. Astron. Soc. Pac., San Francisco, p. 355

Burkert A., Bodenheimer P., 2000, ApJ, 543, 822

Caratti o Garatti A. et al., 2011, A\&A, 526, 4

Chen H., Myers P. C., Ladd E. F., Wood D. O. S., 1995, ApJ, 445, 377

Clarke C. J., Syer D., 1996, MNRAS, 278, L23

Coffey D., Downes T. P., Ray T. P., 2004, A\&A, 419, 593

Draine B. T., Lee H. M., 1984, ApJ, 285, 89

Dullemond C. P., Juhasz A., Pohl A., Sereshti F., Shetty R., Peters T., Commercon B., Flock M., 2012, Astrophysics Source Code Library, record ascl: 1202.015

Dunham M. M. et al., 2013, AJ, 145, 19

Dunham M. M. et al., 2015, ApJS, 220, 26

Dunham M. M., Vorobyov E. I., 2012, ApJ, 747, 52

Duschl W. J., Gail H.-P., Tscharnuter W. M., 1996, A\&A, 312, 624

Enoch M. L., Evans N. J., II, Sargent A. I., Glenn J., 2009, ApJ, 692, 973

Evans N. J., II et al., 2009, ApJS, 181, 321

Fischer W. J., Safron E., Megeath S. T., 2019, ApJ, 872, 183

Flaherty K. M., DeMarchi L., Muzerolle J., Balog Z., Herbst W., Megeath S. T., Furlan E., Gutermuth R., 2016, ApJ, 833, 104

Francis L., Johnstone D., Dunham M. M., Hunter T. R., Mairs S., 2019, ApJ, 871,149

Gardner J. P. et al., 2006, Space Sci. Rev., 123, 485

Hartmann L., Kenyon S. J., 1985, ApJ, 299, 462

Hartmann L., Kenyon S. J., 1996, ARA\&A, 34, 207
Hartmann L., Zhu Z., Calvet N., 2011, 3343, preprint (arXiv:1106.3343)

Hartmann L., Herczeg G., Calvet N., 2016, ARA\&A, 54, 135

Herbig G. H., 1966, Vistas Astron., 8, 109

Herbig G. H., 1977, ApJ, 217, 693

Herbig G. H., 1989, in Reipurth B., ed., European Southern Observatory Conference and Workshop Proceedings Vol. 33, European Southern Observatory Conference and Workshop Proceedings. p. 233

Herbig G. H., 2008, AJ, 135, 637

Herczeg G. J. et al., 2017, ApJ, 849, 14

Hillenbrand L. A., Findeisen K. P., 2015, ApJ, 808, 68

Hubber D. et al., 2011a, Astrophysics Source Code Library, record ascl:1102.010

Hubber D. A., Batty C. P., McLeod A., Whitworth A. P., 2011b, A\&A, 529, 28

Hunter T. R. et al., 2017, ApJ, 837, 6

Jijina J., Myers P. C., Adams F. C., 1999, ApJS, 125, 161

Johnstone D. et al., 2018, ApJ, 854, 21

Johnstone D., Hendricks B., Herczeg G. J., Bruderer S., 2013, ApJ, 765, 133

Kenyon S. J., Hartmann L. W., Strom K. M., Strom S. E., 1990, AJ, 99, 869

Kenyon S. J., Kolotilov E. A., Ibragimov M. A., Mattei J. A., 2000, ApJ, 531,1028

Kóspál Á. et al., 2011, A\&A, 527, 9

Kóspál Á., Ábrahám P., Prusti T., Acosta-Pulido J., Hony S., Moór A., Siebenmorgen R., 2007, A\&A, 470, 211

Kuffmeier M., Frimann S., Jensen S. S., Haugbølle T., 2018, MNRAS, 475, 2642

Larson R. B., 1981, R. Astron. Soc., 194, 809

Lenzuni P., Gail H.-P., Henning T., 1995, ApJ, 447, 848

Liu H. B. et al., 2016, Sci. Adv., 2, e1500875

Lodato G., Clarke C. J., 2004, MNRAS, 353, 841

Lorenzetti D. et al., 2006, A\&A, 453, 579

MacFarlane B. A., Stamatellos D., 2017, MNRAS, 472, 3775

Mairs S. et al., 2017, ApJ, 849, 22

Mairs S. et al., 2018, Astron. Telegram, 11583, 1

Mathis J. S., Rumpl W., Nordsieck K. H., 1977, ApJ, 217, 425

Mercer A., Stamatellos D., 2017, MNRAS, 465, 2

Millan-Gabet R. et al., 2006, ApJ, 641, 547

Min M., Dullemond C. P., Dominik C., de Koter A., Hovenier J. W., 2009, A\&A, 497, 155

Morris J. P., Monaghan J. J., 1997, J. Comput. Phys., 136, 41

Myers P. C., Ladd E. F., 1993, ApJ, 413, L47

Natta A., Testi L., 2004, in Johnstone D., Adams F. C., Lin D. N. C., Neufeeld D. A., Ostriker E. C., eds, ASP Conf. Ser. Vol. 323, Star Formation in the Interstellar Medium: In Honor of David Hollenbach. Astron. Soc. Pac., San Francisco, p. 279

Offner S. S. R., Klein R. I., McKee C. F., Krumholz M. R., 2009, ApJ, 703, 131

Ossenkopf V., Henning T., 1994, A\&A, 291, 943

Peña C. C., Naylor T., Morrell S., 2019, MNRAS, 486, 4590

Pfalzner S., 2008, A\&A, 492, 735

Pfalzner S., Tackenberg J., Steinhausen M., 2008, A\&A, 487, L45

Price D. J., 2007, Publ. Astron. Soc. Aust., 24, 159

Pudritz R. E., Ouyed R., Fendt C., Brandenburg A., 2007, in Reipurth B., Jewitt D., Kkeil K., eds, Protostars and Planets V. Univ. Arizona Press, Tucson, 277

Rebull L. M. et al., 2015, AJ, 150, 37

Reipurth B., Aspin C., 2004, ApJ, 608, L65

Rigon L., Scholz A., Anderson D., West R., 2017, MNRAS, 465, 3889

Robitaille T. P., 2010, A\&A, 520, 3

Robitaille T. P., 2011, A\&A, 536, 17

Sadavoy S. I. et al., 2014, ApJ, 787, 6

Safron E. J. et al., 2015, ApJ, 800, 6

Scholz A., Froebrich D., Wood K., 2013, MNRAS, 430, 2910

Shakura N. I., Sunyaev R. A., 1973, A\&A, 24, 337

Shu F. H., 1977, ApJ, 214, 488

Shu F. H., Adams F. C., Lizano S., 1987, ARA\&A, 25, 23 
Sipos N., Ábrahám P., Acosta-Pulido J., Juhász A., Kóspál Á, Kun M., Moór A., Setiawan J., 2009, A\&A, 507, 881

Stahler S. W., Shu F. H., Taam R. E., 1980a, ApJ, 241, 637

Stahler S. W., Shu F. H., Taam R. E., 1980b, ApJ, 242, 226

Stamatellos D., 2003, Ph.D. Thesis, Cardiff University, UK

Stamatellos D., Whitworth A. P., 2003, A\&A, 407, 941

Stamatellos D., Whitworth A. P., 2005, A\&A, 439, 153

Stamatellos D., Whitworth A. P., André P., Ward-Thompson D., 2004, A\&A, 420, 1009

Stamatellos D., Whitworth A. P., Bisbas T., Goodwin S., 2007, A\&A, 475, 37

Stamatellos D., Whitworth A. P., Hubber D. A., 2011, ApJ, 730, 32

Stamatellos D., Whitworth A. P., Hubber D. A., 2012, MNRAS, 427, 1182

Vorobyov E. I., Basu S., 2005, ApJ, 633, L137
Vorobyov E. I., Basu S., 2006, ApJ, 650, 956

Vorobyov E. I., Basu S., 2010, ApJ, 714, L133

Whitney B. A., Wood K., Bjorkman J. E., Wolff M. J., 2003a, ApJ, 591, 1049

Whitney B. A., Wood K., Bjorkman J. E., Cohen M., 2003b, ApJ, 598, 1079

Yoo H. et al., 2017, ApJ, 849, 10

Zhu Z., Hartmann L., Gammie C., 2009a, ApJ, 694, 1045

Zhu Z., Hartmann L., Gammie C., McKinney J. C., 2009b, ApJ, 701, 620

Zhu Z., Hartmann L., Gammie C. F., Book L. G., Simon J. B., Engelhard E., 2010a, ApJ, 713, 1134

Zhu Z., Hartmann L., Gammie C., 2010b, ApJ, 713, 1143

This paper has been typeset from a $\mathrm{T}_{\mathrm{E}} \mathrm{X} / \mathrm{L} \mathrm{T} \mathrm{E} \mathrm{X}$ file prepared by the author. 